\title{
Impact of currently prescribed lipid-lowering drugs on plasma PCSK9 concentration: single or in combination study in rats
}

\author{
Yan Zhang ${ }^{\dagger}$, Jun Liư ${ }^{\dagger}$, Sha Li, Rui-Xia Xu, Jing Sun and Jian-Jun Li ${ }^{*}$
}

\begin{abstract}
Background: An emerging data suggested a significant impact of statins on PCSK9 concentration, while the rapid impacts of other lipid-lowering drugs such as ezetimibe and xuezhikang alone or in combination on PCSK9 and lipid profile have not been assessed. This study aims to investigate whether an enhanced PCSK9 concentration by single or combined therapy of lipid-lowering drugs currently used precedes the changes of lipid profile in rats.

Methods: Sixty-three rats were randomly divided into six groups and orally administrated with placebo $(N=13)$, ezetimibe 10 mg/kg daily, Xuezhikang $1200 \mathrm{mg} / \mathrm{kg}$ daily, ezetimibe $10 \mathrm{mg} / \mathrm{kg}$ plus Xuezhikang $1200 \mathrm{mg} / \mathrm{kg}$ daily, pitavastatin $10 \mathrm{mg} / \mathrm{kg}$ daily or pitavastatin $10 \mathrm{mg} / \mathrm{kg}$ plus ezetimibe $10 \mathrm{mg} / \mathrm{kg}$ daily for 3 days $(\mathrm{N}=10$ for each group respectively). Blood samples were obtained at day 3 after orally administration. Plasma PCSK9 levels were determined by ELISA and lipid profile were measured by enzymatic assay.

Results: Ezetimibe, Xuezhikang and pitavastatin alone and Xuezhikang plus ezetimibe as well as pitavastatin plus ezetimibe increased PCSK9 levels by 124\%, 56\%, 111\%, 63\% and 204\% respectively ( $p<0.05$ compared with placebo). However, Xuezhikang plus ezetimibe did not enhance greater PCSK9 levels compared to monotherapy. Ezetimibe and pitavastatin in combination induced higher PCSK9 levels than pitavastatin monotherapy or co-therapy with ezetimibe plus Xuezhikang. There was no significant difference between any groups with regard to lipid profile levels at day $3(P>0.05)$.
\end{abstract}

Conclusions: Elevated PCSK9 concentration by ezetimibe, Xuezhikang and pitavastatin alone or in combination was found prior to the alterations of lipid profile in rats. Combination of Xuezhikang and ezetimibe significantly attenuated increase in PCSK9 compared to ezetimibe plus pitavastatin, suggesting that the former combination may be better than the latter in future clinical application.

Keywords: PCSK9, Statin, Lipid profile, Rat

\section{Introduction}

Proprotein convertase subtilisin/kexin type 9 (PCSK9) is a secreted protease synthesized mainly in the liver, which binds directly to the epidermal growth factor repeat A of the low-density lipoprotein receptor (LDLR) and subsequently targets it for degradation [1]. This process results in increased serum LDL-cholesterol (LDL-C) levels in the circulation [2]. Gain-of-function mutations in the

\footnotetext{
*Correspondence: lijianjun938@yahoo.com

${ }^{\dagger}$ Equal contributors

Division of Dyslipidemia, State Key Laboratory of Cardiovascular Disease, Fu Wai Hospital, National Center for Cardiovascular Diseases, Chinese Academy

of Medical Sciences, Peking Union Medical College, BeiLiShi Road 167, Beijing 100037, China
}

(c) 2014 Zhang et al.; licensee BioMed Central Ltd. This is an Open Access article distributed under the terms of the Creative Commons Attribution License (http://creativecommons.org/licenses/by/2.0), which permits unrestricted use, distribution, and reproduction in any medium, provided the original work is properly credited. The Creative Commons Public Domain Dedication waiver (http://creativecommons.org/publicdomain/zero/1.0/) applies to the data made available in this article, unless otherwise stated.

PCSK9 gene are associated with autosomal dominant hypercholesterolemia and premature cardiovascular disease $[3,4]$. Conversely, loss-of-function mutations of the PCSK9 gene linked to low LDL-C levels and a dramatically reduced risk for coronary heart disease [5]. Therefore, PCSK9 has been considered as another target for dyslipidemia and atherosclerosis [6].

Recently, several studies indicated that statins (HMGCoA reductase inhibitors) could upregulate PCSK9 levels in humans [7-9], which may attenuate the beneficial effects of statins [10]. It was recently postulated that statins increase the activity of sterol regulator elementbinding protein 2 (SREBP-2), which is a transcription 
factor that activates both the LDLR and PCSK9 genes [11], and eventually induces elevated expression and secretion of PCSK9 protein. In addition, other common prescribed lipid-lowering drugs, ezetimibe, Xuezhikang are widely used as alone or in combination for the management of dyslipidemia. For example, ezetimibe is an inhibitor of intestinal cholesterol absorption, which could decrease LDL-C by approximately $20 \%$ when administered alone [12] and when combined with statin could achieve $15 \%$ more decrease in LDL-C concentrations compared with statin alone [13]. However, a few reports about whether ezetimibe could exert similar effects on PCSK9 as statins are inconsistent [14,15].

Extracts of red yeast rice have been widely used for therapy of patients with cardiovascular disorders in China for centuries. Xuezhikang, a partially purified extract of fermented red yeast rice, contains 13 kinds of natural statins (high amounts of lovastatin), unsaturated fatty acids, ergosterol, flavonoids, and some other components [16,17]. A systematic review demonstrated that Xuezhikang exerted significant lipid-lowering effects in the treatment of hyperlipidemia [18]. Recently, clinical studies indicated that Xuezhikang could be used as an alternative therapy for patients intolerant of statin [19]. However, there are limited data to evaluate the impact of Xuezhikang on the PCSK9 levels. Although previous studies have implied that lipid-lowering drugs have short-term effects on PCSK9 in humans [20], study investigating whether the impact of currently used lipidlowering drugs on PCSK9 levels prior to the changes of lipid profile is still limited. Moreover, the impacts of lipid-lowering drugs in combination on PCSK9 has less been evaluated till now.

Therefore, the aim of the present study was to evaluate: 1) whether the increase of PCSK9 concentration is prior to the decrease of LDL-C levels; 2) the rapid effects of different lipid-lowering drugs on PCSK9 concentration; 3) the different impacts of lipid-lowering drugs in combination on PCSK9 concentration.

\section{Materials and methods}

\section{Animal preparation}

Male Sprague-Dawley rats, weighing 180-220 g, were fed in a temperature-conditioned $\left(22-24^{\circ} \mathrm{C}\right)$ room with alternating $12 \mathrm{~h}$ light/dark cycles and allowed them free access to food and water for 3 days to get familiar with the environment. At the start of the study, 63 rats were randomly assigned to six groups: (1) placebo group ( $\mathrm{N}=13$, orally administrated with normal saline), (2) ezetimibe group $(\mathrm{N}=10$, orally administrated with ezetimibe $10 \mathrm{mg} / \mathrm{kg}$ daily), (3) Xuezhikang group ( $\mathrm{N}=10$, orally administrated with Xuezhikang $1200 \mathrm{mg} / \mathrm{kg}$ daily), (4) ezetimibe plus Xuezhikang group $(\mathrm{N}=10$, orally administrated with ezetimibe $10 \mathrm{mg} / \mathrm{kg}$ plus Xuezhikang
$1200 \mathrm{mg} / \mathrm{kg}$ daily), (5) pitavastatin group $(\mathrm{N}=10$, orally administrated with pitavastatin $10 \mathrm{mg} / \mathrm{kg}$ daily), and (6) pitavastatin plus ezetimibe group $(\mathrm{N}=10$, orally administrated with pitavastatin $10 \mathrm{mg} / \mathrm{kg}$ plus ezetimibe $10 \mathrm{mg} / \mathrm{kg}$ daily). This study was conducted in accordance with the Guide for the Care and Use of Laboratory Animals published by the National Institutes of Health, and all experimental procedures and protocols were approved by the Care of Experimental Animals Committee of Fuwai Hospital, Chinese Academy of Medical Sciences and Peking Union Medical College.

\section{Blood sample and analysis}

After 3 days orally administration, prior to sacrifice, $2 \mathrm{ml}$ fasting blood were collected from the inferior vena cava and transferred to K2 EDTA tubes. Immediately after blood sampling, the animals were euthanized by an overdose of pentobarbital sodium. The blood samples were centrifuged, and the plasma was stored at $-80^{\circ} \mathrm{C}$ until the analyses were performed. Concentrations of serum total cholesterol (TC), triglycerides (TG), high density lipoprotein cholesterol (HDL-C) and LDL-C were determined on an automatic biochemistry analyzer (Hitachi 7150, Tokyo, Japan). Plasma PCSK9 concentrations were measured using a high-sensitivity, quantitative sandwich enzyme immunoassay (Quantikine ELISA, R\&D Systems Europe Ltd). The lower limit of detection was $0.096 \mathrm{ng} / \mathrm{ml}$.

\section{Statistical analysis}

Continuous variables were expressed as mean \pm standard deviation (SD) and categorical variables were presented as frequent count and percentages. The comparison of inter-group analysis of PCSK9 and lipid profile levels was performed by unpaired two-tailed t-test and one way ANOVA. A P value of less than 0.05 was considered statistical significance. The SPSS 19.0 statistical software package (SPSS Inc., Chicago, IL, USA) was used for all of the statistical analysis.

\section{Results}

\section{The changes of lipid profile}

As shown in Table 1, there was no significant difference in lipid profile among rats receiving placebo, ezetimibe, Xuezhikang, pitavastatin, ezetimibe plus Xuezhikang, or pitavastatin plus ezetimibe at day $3(\mathrm{p}>0.05)$.

\section{Rapid effects of lipid-lowering drugs on plasma PCSK9 concentrations}

As shown in Figures 1 and 2, at day 3, PCSK9 levels increased from $296.29 \pm 112.95 \mathrm{ng} / \mathrm{ml}$ to $664.76 \pm 331.73 \mathrm{ng} /$ $\mathrm{ml}$ in rats receiving ezetimibe $10 \mathrm{mg} / \mathrm{kg}$ daily treatment (124\%; p < 0.05 vs. placebo). In rats treated with Xuezhikang $1200 \mathrm{mg} / \mathrm{kg}$ daily, PCSK9 levels increased from 
Table 1 Laboratory data

\begin{tabular}{lllllll}
\hline Parameters & $\begin{array}{l}\text { Placebo } \\
(\mathbf{N}=13)\end{array}$ & $\begin{array}{l}\text { Ezetimibe } \\
(\mathbf{N}=10)\end{array}$ & $\begin{array}{l}\text { Xuezhikang } \\
(\mathbf{N}=10)\end{array}$ & $\begin{array}{l}\text { Xuezhikang + ezetimibe } \\
(\mathbf{N}=10)\end{array}$ & $\begin{array}{l}\text { Pitavstatin } \\
(\mathbf{N}=10)\end{array}$ & $\begin{array}{l}\text { Pitavstatin + ezetimibe } \\
(\mathbf{N}=10)\end{array}$ \\
\hline PCSK9 ng/ml & $296.29 \pm 112.95$ & $664.76 \pm 331.73^{*}$ & $461.20 \pm 124.96^{*}$ & $482.86 \pm 130.29^{*}$ & $626.16 \pm 221.48^{*}$ & $901.35 \pm 235.56^{* \# \Delta}$ \\
TG mmol/l & $0.76 \pm 0.72$ & $0.51 \pm 0.17$ & $1.38 \pm 0.12$ & $1.26 \pm 0.29$ & $0.44 \pm 0.12$ & $0.91 \pm 0.18$ \\
TC mmol/l & $2.13 \pm 0.26$ & $2.44 \pm 0.40$ & $2.25 \pm 0.37$ & $2.19 \pm 0.40$ & $2.50 \pm 0.40$ & $2.39 \pm 0.33$ \\
HDL-C mmol/l & $1.04 \pm 0.29$ & $1.31 \pm 0.29$ & $1.04 \pm 0.29$ & $0.94 \pm 0.20$ & $1.25 \pm 0.27$ & $1.22 \pm 0.27$ \\
LDL-C mmol/l & $0.53 \pm 0.14$ & $0.51 \pm 0.15$ & $0.45 \pm 0.07$ & $0.42 \pm 0.04$ & $0.64 \pm 0.18$ & $0.48 \pm 0.09$ \\
\hline
\end{tabular}

Data are expressed as means \pm SD. ${ }^{*} p<0.05$ versus placebo group. ${ }^{\#} p<0.05$ versus pitavastatin group. ${ }^{\Delta} p<0.05$ versus Xuezhikang + ezetimibe group. PCSK9 = proprotein convertase subtilisin/kexin type 9; TG = triglycerides; $\mathrm{TC}=$ total cholesterol; $\mathrm{HDL}-\mathrm{C}=$ high density lipoprotein cholesterol; $\mathrm{LDL}-\mathrm{C}=\mathrm{low}$ density lipoprotein cholesterol.

$296.29 \pm 112.95 \mathrm{ng} / \mathrm{ml}$ to $461.20 \pm 124.96 \mathrm{ng} / \mathrm{ml}$ (56\% increase, $\mathrm{p}<0.05$ vs. placebo). In rats with pitavastatin $10 \mathrm{mg} / \mathrm{kg}$ daily treatment, PCSK9 increased from $296.29 \pm$ $112.95 \mathrm{ng} / \mathrm{ml}$ to $626.16 \pm 221.48 \mathrm{ng} / \mathrm{ml}$ (111\% increase, $\mathrm{p}<0.05$ vs. placebo). The combination of ezetimibe $10 \mathrm{mg} /$ $\mathrm{kg}$ daily plus Xuezhikang $1200 \mathrm{mg} / \mathrm{kg}$ daily resulted in an increase of PCSK9 levels by $63 \%(296.29 \pm 112.95$ to $482.86 \pm 130.29 \mathrm{ng} / \mathrm{ml})$. Pitavastatin $10 \mathrm{mg} / \mathrm{kg}$ daily and ezetimibe $10 \mathrm{mg} / \mathrm{kg}$ daily in combination resulted in an increase of PCSK 9 by $204 \%$ (296.29 \pm 112.95 to $901.35 \pm$ $235.56 \mathrm{ng} / \mathrm{ml} ; \mathrm{p}<0.05$ vs. placebo).

\section{Combination of ezetimibe plus Xuezhikang, ezetimibe plus pitavastatin on plasma PCSK9 concentrations}

As shown in Table 1, at day 3, in combination of ezetimibe and Xuezhikang did not induce greater increase of PCSK9 concentrations than monotherapy with either agent $(\mathrm{p}>0.05)$. However, as shown in Figures 3 and 4, the PCSK9 concentrations among rats treated with ezetimibe plus pitavastatin was much higher than monotherapy with pitavastatin or the co-therapy of ezetimibe plus Xuezhikang $(\mathrm{p}<0.05)$.

\section{Discussion}

The current experiment explored the rapid effects of the 3 currently prescribed lipid-lowering drugs, pitavastatin, ezetimibe and Xuezhikang, alone and in combination, on PCSK9 and lipid profile concentrations in rats in a very short period. The main finding of this study is that ezetimibe, Xuezhikang and pitavastatin alone and Xuezhikang plus ezetimibe as well as pitavastatin plus ezetimibe, all could significantly increase the PCSK9 levels at day 3 , when the lipid profile had no obvious decreases. In addition, combination of ezetimibe $10 \mathrm{mg} / \mathrm{kg}$ and Xuezhikang $1200 \mathrm{mg} / \mathrm{kg}$ daily did not further enhance PCSK9 levels compared to the monotherapy, while ezetimibe $10 \mathrm{mg} / \mathrm{kg}$ and pitavastatin $10 \mathrm{mg} / \mathrm{kg}$ daily in combination induced higher PCSK9 levels than monotherapy with pitavastatin or co-therapy with ezetimibe plus Xuezhikang.

It has been well established that PCSK9 promotes the degradation of LDLR and could limit the beneficial effects of lipid-lowering drugs [1]. Several studies indicated that lipid-lowering drugs could elevate plasma PCSK9 concentrations and may have rapid impact on PCSK9 levels [20]. In our present study, we confirmed previous studies and provided novel additional important information regarding the lipid-lowering drugs on plasma PCSK9. This study, for the first time, indicated that the enhancement of PCSK9 levels preceded the changes of lipid profile. Therefore, it is reasonable to deduce that patients with dyslipidemia may get more benefits from PCSK9 inhibitors, such as monoclonal antibody [21], if it be used before the application of lipid-lowering drugs in clinical practice. Although commonly prescribed lipid-lowering drugs may up-regulate both the LDLR and PCSK9 genes by activating SREBP-2 [11], the PCSK9 gene might respond much earlier than

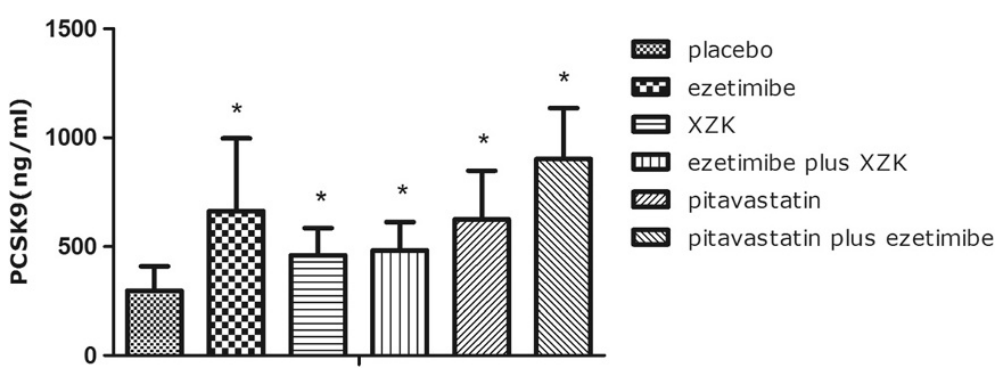

Figure 1 PCSK9 levels during the treatment with placebo, ezetimibe, Xuezhikang, Xuezhikang plus ezetimibe, pitavastatin and pitavastatin plus ezetimibe. ${ }^{*} p<0.05$ versus placebo group. XZK: Xuezhikang. 


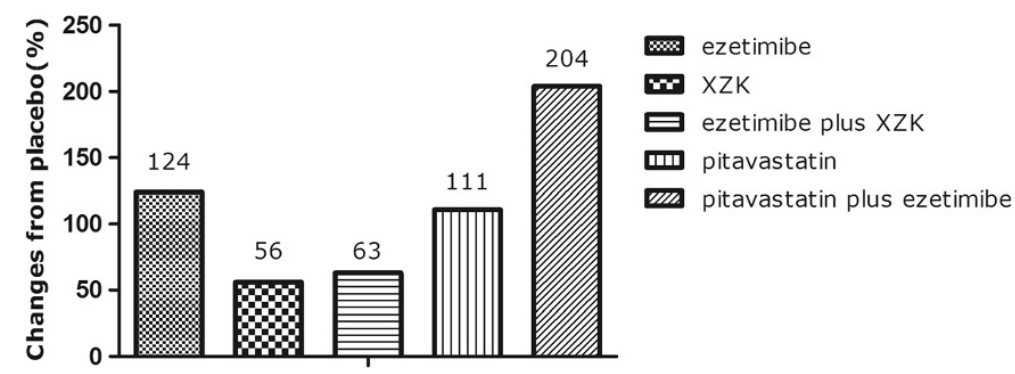

Figure 2 Mean percentage changes from placebo in PCSK9 levels during the treatment with ezetimibe, Xuezhikang, Xuezhikang plus ezetimibe, pitavastatin and pitavastatin plus ezetimibe. XZK: Xuezhikang.

the LDLR gene, that may partly of the reason why the changes of PCSK9 levels preceded the lipid profile.

Currently, combination of two different lipid-lowering drugs is frequently prescribed in controlling dyslipidemia. Statin plus ezemtibe in combination is the most commonly used style. In recent years, several researchers have described the combination of statin and ezetimibe on the increase of PCSK9 levels in humans. A recent study conducted by Davignon et al. [22] demonstrated that patients treated with statins alone had a $45 \%$ increase in PCSK9 levels and those treated with statin plus ezetimibe showed an approximately $77 \%$ increase in PCSK9 concentrations. In our study, the PCSK9 levels increased by $111 \%$ in rats administrated with pitavastatin $10 \mathrm{mg} / \mathrm{kg}$ daily, while added ezetimibe $10 \mathrm{mg} / \mathrm{kg}$ daily to pitavastatin, the PCSK9 levels enhanced by $204 \%$. However, little was known about the reason why ezetimibe causes a further increase in PCSK9 levels. Since ezetimibe exerts its effect by binding to Niemann-Pick C1-Like 1, and thereby inhibiting intestinal cholesterol absorption and subsequently results in reduced hepatic cholesterol [23], it may be possible that this process is accompanied by a feedback mechanism on transcription factor SREBP-2. Furthermore, in mice models, Brandon Ason et al. [15] applied qRT-PCR to analyze the expression of 361 genes involved in hepatic lipid metabolism and found that many genes within the SREBP-2 pathway were induced following ezetimibe treatment (2.5-fold average induction relative to control) and were even further induced by ezetimibe plus rosuvastatin combination treatment (11-fold average induction relative to control). This study provides theoretical evidences about the impact of ezetimibe on plasma PCSK9 levels. In contrast, Heiner K. Berthold et al. [14] indicated that when added to simvastatin, ezetimibe does not cause an incremental increase in PCSK9 concentrations, which conflicted with our observations. However, this study explored the 14 days effect of ezetimibe-statin in combination on plasma PCSK9 levels in humans, and different kinds of statins were used in the two studies. Even so, for the sake of exploring a thorough explanation of the mechanism, further studies are warranted.

In our study, we also observed that combination of ezetimibe $10 \mathrm{mg} / \mathrm{kg}$ and Xuezhikang $1200 \mathrm{mg} / \mathrm{kg}$ daily did not bring a greater increase in PCSK9 levels than monotherapy, and the PCSK9 levels was significantly lower than co-therapy with ezetimibe plus pitavastatin. Therefore, for patients who need an intensive lipidlowering therapy, the combination of Xuezhikang and ezetimibe would be a better alternative therapy. This combination could be a better strategy not only for exerting good lipid-lowering effect on LDL-C, but also for mitigating the adverse effects of lipid-lowering drugs on PCSK9 in controlling dyslipidemia and atherosclerosis. At the present time, with regarding to the mechanism by which ezetimibe plus Xuezhikang causes an

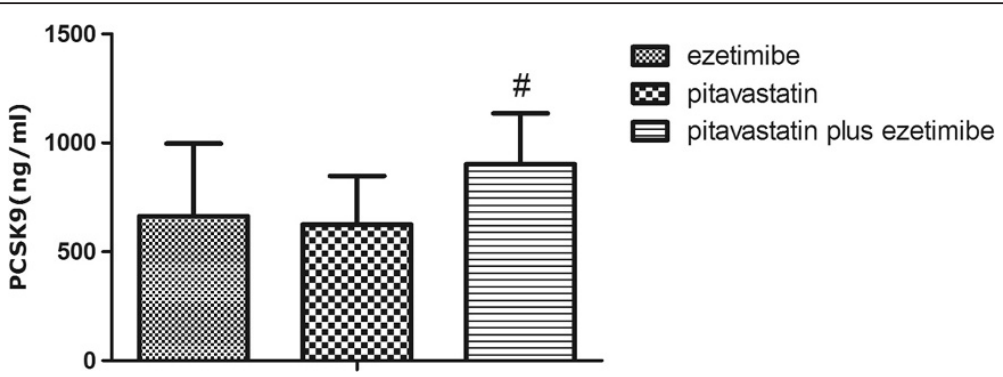

Figure 3 PCSK9 levels during the treatment with ezetimibe, pitavastatin and pitavastatin plus ezetimibe. ${ }^{\#} p<0.05$ versus pitavastatin group. XZK: Xuezhikang. 


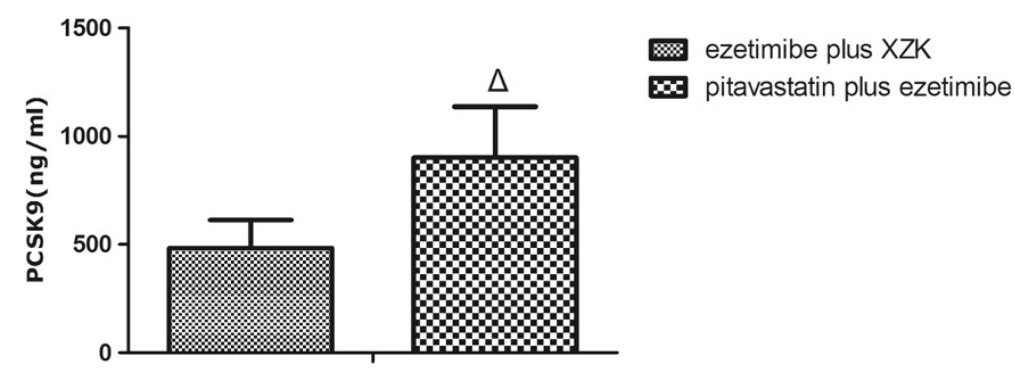

Figure 4 PCSK9 levels during the treatment with Xuezhikang plus ezetimibe and pitavastatin plus ezetimibe. ${ }^{\Delta} p<0.05$ versus Xuezhikang plus ezetimibe group. XZK: Xuezhikang.

attenuated increase in PCSK9 levels compared to monotherapy and co-therapy with ezetimibe plus pitavastatin is unclear, but it may likely associated with the chemical constituents of Xuezhikang. Xuezhikang, a Chinese traditional medicine, is widely used as a lipid-lowering drug, and its main components contain lovastatin, as well as other useful substances [16-18]. Recent studies including our data have shown that Xuezhikang has many pleiotropic effects, which could effectively modify not only lipid profile but also inflammatory markers [24]. The interactions among different constituents of Xuezhikang and ezetimibe may influence the effect on PCSK9 levels, but clinical trials and molecular mechanisms are needed in the further studies.

For the past decade, PCSK9 has gained tremendous attention. It is well-established that statin treatment elevates plasma PCSK9 levels and, on the contrary, inhibition of PCSK9 function enhances the lipid-lowering effects of statins $[25,26]$. Among the various PCSK9 inhibitors, human data are available for monoclonal antibodies against PCSK9 of which the two most advanced are SAR236553/REGN727 and AMG 145. In a 12-week phase II study of patients with LDL-C levels $\geq 100 \mathrm{mg} / \mathrm{dl}$ on a stable dose of atorvastatin $(10,20$, or $40 \mathrm{mg} /$ day), add-on of SAR236553/REGN727 (doses 50-150 mg) administered subcutaneously every 2 weeks resulted in LDL-C reductions of 40-72\% [27]. The LAPLACE-TIMI trial evaluated patients with documented hypercholesterolemia, while on a statin with or without ezetimibe treatment. The data had shown reductions of LDL-C by $41.8 \%$ to $66.1 \%$ when administrated AMG 145 at 70,105 and $140 \mathrm{mg}$ every 2 weeks, with acceptable safety and tolerability [21]. Combining our findings and previous studies together may suggest that, not only statins, but also ezetimibe and Xuezhikang could increase plasma PCSK9 levels, and this effects occurred before the changes of lipid-profile, which strongly suggest that PCSK9 inhibitors should be combined with the currently prescribed lipid-lowering drugs to result in further LDL-C lowering effect, and it would be better to be used prior to lipid-lowering drugs. Moreover, our data also provides additional information with respect to combination of ezetimibe plus Xuezhikang may be a better choice than ezetimibe plus pitavastatin in clinical practice.

There are several limitations in the present study. First, we did not observe the long-term effect of different lipid-lowering drugs, alone and in combination, on plasma PCSK9 and lipid-profile concentrations. Second, we did not explore the molecular mechanisms of the phenomenon. Hence, further studies are needed.

\section{Competing interests}

The authors declare that they have no competing interests.

\section{Authors' contributions}

ZY and LJ completed the project, and analyzed the data, and wrote the manuscript. $\sqcup-J$ established the study, interpreted the data, and contributed to reviewing/editing the manuscript. LS, XR-X, and SJ contributed to assay and analyzing the data. All authors read and approved the final manuscript.

\section{Acknowledgements}

This work was partly supported by National Natural Scientific Foundation (81070171, 81241121), Specialized Research Fund for the Doctoral Program of Higher Education of China (20111106110013), Capital Special Foundation of Clinical Application Research (Z121107001012015), Capital Health

Development Fund (2011400302), and Beijing Natural Science Foundation (7131014) awarded by Dr. Jian-Jun Li, MD, PhD.

Received: 12 January 2014 Accepted: 10 February 2014 Published: 18 February 2014

\section{References}

1. Seidah NG, Prat A: The biology and therapeutic targeting of the proprotein convertases. Nat Rev Drug Discov 2012, 11:367-383.

2. Horton JD, Cohen JC, Hobbs HH: PCSK9: a convertase that coordinates LDL catabolism. J Lipid Res 2009, 50(Suppl):S172-S177.

3. Abifadel $M$, Varret $M$, Rabès JP, Allard D, Ouguerram K, Devillers M, Cruaud C, Benjannet S, Wickham L, Erlich D, Derré A, Villéger L, Farnier M, Beucler I, Bruckert E, Chambaz J, Chanu B, Lecerf JM, Luc G, Moulin P, Weissenbach J, Prat A, Krempf M, Junien C, Seidah NG, Boileau C: Mutations in PCSK9 cause autosomal dominant hypercholesterolemia. Nat Genet 2003, 34:154-156.

4. Wu NQ, Guo YL, Xu RX, Liu J, Zhu CG, Jiang LX, Li JJ: Acute myocardial infarction in an 8-year old male child with homozygous familiar hypercholesterolemia: laboratory findings and response to lipid-lowering drugs. Clin Lab 2013, 59:901-907.

5. Cohen JC, Boerwinkle E, Mosley TH Jr, Hobbs HH: Sequence variations in PCSK9, low LDL, and protection against coronary heart disease. $N$ Engl J Med 2006, 354:1264-1272. 
6. Urban D, Pöss J, Böhm M, Laufs U: Targeting the proprotein convertase subtilisin/kexin type 9 for the treatment of dyslipidemia and atherosclerosis. J Am Coll Cardiol 2013, 62:1401-1408.

7. Welder G, Zineh I, Pacanowski MA, Troutt JS, Cao G, Konrad RJ: High-dose atorvastatin causes a rapid sustained increase in human serum PCSK9 and disrupts its correlation with LDL cholesterol. J Lipid Res 2010, 5:2714-2721.

8. Awan Z, Seidah NG, MacFadyen JG, Benjannet S, Chasman DI, Ridker PM, Genest J: Rosuvastatin, proprotein convertase Subtilisin/Kexin type 9 concentrations, and LDL cholesterol response: the JUPITER Trial. Clin Chem 2012, 58:183-189.

9. Raal F, Panz V, Immelman A, Pilcher G: Elevated PCSK9 levels in untreated patients with heterozygous or homozygous familial hypercholesterolemia and the response to high-dose statin therapy. J Am Heart Assoc 2013, 2:e000028.

10. Careskey HE, Davis RA, Alborn WE, Troutt JS, Cao G, Konrad RJ: Atorvastatin increases human serum levels of proprotein Convertase subtilisin/kexin type 9. J Lipid Res 2008, 49:394-398.

11. Dubuc G, Chamberland A, Wassef H, Davignon J, Seidah NG, Bernier L, Prat A: Statins upregulate PCSK9, the gene encoding the proprotein convertase hypercholesterolemia. Arterioscler Thromb Vasc Biol 2004, 24:1454-1459.

12. Taylor AJ, Villines TC, Stanek EJ, Devine PJ, Griffen L, Miller M, Weissman NJ, Turco M: Extended-release niacin or ezetimibe and carotid intima-media thickness. N Engl J Med 2009, 361:2113-2122.

13. Morrone D, Weintraub WS, Toth PP, Hanson ME, Lowe RS, Lin J, Shah AK Tershakovec AM: Lipid-altering efficacy of ezetimibe plus statin and statin monotherapy and identification of factors associated with treatment response: a pooled analysis of over 21,000 subjects from 27 clinical trials. Atherosclerosis 2012, 223:251-261.

14. Berthold HK, Seidah NG, Benjannet S, Gouni-Berthold I: Evidence from a randomized trial that simvastatin, but not ezetimibe, upregulates circulating PCSK9 levels. PLoS One 2013, 8:e60095.

15. Ason B, Tep S, Davis HR Jr, Xu Y, Tetzloff G, Galinski B, Soriano F, Dubinina N, Zhu L, Stefanni A, Wong KK, Tadin-Strapps M, Bartz SR, Hubbard B, Ranalletta M, Sachs AB, Flanagan WM, Strack A, Kuklin NA: Improved efficacy for ezetimibe and rosuvastatin by attenuating the induction of PCSK9. J Lipid Res 2011, 52:679-687.

16. Lu Z, Kou W, Du B, Wu Y, Zhao S, Brusco OA, Morgan JM, Capuzzi DM, Chinese Coronary Secondary Prevention Study Group, Li S: Effect of Xuezhikang, an extract from red yeast Chinese rice, on coronary events in a Chinese population with previous myocardial infarction. Am J Cardiol 2008, 101:1689-1693.

17. Li JJ, Lu ZL, Kou WR, Chen Z, Wu YF, Yu XH, Zhao YC, Chinese Coronary Secondary Prevention Study Group: Impact of Xuezhikang on coronary events in hypertensive patients with previous myocardial infarction from the China Coronary Secondary Prevention Study (CCSPS). Ann Med 2010, 42:231-240.

18. Liu ZL, Liu JP, Zhang AL, Wu Q, Ruan Y, Lewith G, Visconte D: Chinese herbal medicines for hypercholesterolemia. Cochrane Database Syst Rev 2011, 7, CD008305.

19. Becker DJ, Gordon RY, Halbert SC, French B, Morris PB, Rader DJ: Red yeast rice for dyslipidemia in statin-intolerant patients: a randomized trial. Ann Intern Med 2009, 150:830-839.

20. Guo YL, Liu J, Xu RX, Zhu CG, Wu NQ, Jiang LX, Li JJ: Short-term impact of low-dose atorvastatin on serum proprotein convertase Subtilisin/Kexin type 9. Clin Drug Investig 2013, 33:877-883.

21. Giugliano RP, Desai NR, Kohli P, Rogers WJ, Somaratne R, Huang F, Liu T, Mohanavelu S, Hoffman EB, McDonald ST, Abrahamsen TE, Wasserman SM, Scott R, Sabatine MS, LAPLACE-TIMI 57 Investigators: Efficacy, safety, and tolerability of a monoclonal antibody to proprotein convertase subtilisin/ kexin type 9 in combination with a statin in patients with hypercholesterolaemia (LAPLACE-TIMI 57): a randomised, placebo-controlled, dose-ranging, phase 2 study. Lancet 2012, 380:2007-2017.

22. Davignon J, Dubuc G: Statins and ezetimibe modulate plasma proprotein convertase subtilisin kexin-9 (PCSK9) levels. Trans Am Clin Climatol Assoc 2009, 120:163-173

23. Bays HE, Neff D, Tomassini JE, Tershakovec AM: Ezetimibe: cholesterol lowering and beyond. Expert Rev Cardiovasc Ther 2008, 6:447-470
24. Li JJ, Wang Y, Nie SP, Li YS, Huang Y, Hui RT: Xuezhikang, an extract of cholestin, decreases plasma inflammatory markers and endothelin-1, improve exercise-induced ischemia and subjective feeling in patients with cardiac syndrome X. Int J Cardiol 2007, 122:82-84.

25. Stein EA, Gipe D, Bergeron J, Gaudet D, Weiss R, Dufour R, Wu R, Pordy R: Effect of a monoclonal antibody to PCSK9, REGN727/SAR236553, to reduce low-density lipoprotein cholesterol in patients with heterozygous familial hypercholesterolaemia on stable statin dose with or without ezetimibe therapy: a phase 2 randomised controlled trial. Lancet 2012, 380:29-36.

26. Poirier S, Mayer G: The biology of PCSK9 from the endoplasmic reticulum to lysosomes: new and emerging therapeutics to control low-density lipoprotein cholesterol. Drug Des Devel Ther 2013, 4:1135-1148.

27. Stein EA, Mellis S, Yancopoulos GD, Stahl N, Logan D, Smith WB, Lisbon E, Gutierrez M, Webb C, Wu R, Du Y, Kranz T, Gasparino E, Swergold GD: Effect of a monoclonal antibody to PCSK9 on LDL cholesterol. N Engl J Med 2012, 366:1108-1118.

doi:10.1186/1476-511X-13-35

Cite this article as: Zhang et al:: Impact of currently prescribed lipidlowering drugs on plasma PCSK9 concentration: single or in combination study in rats. Lipids in Health and Disease 2014 13:35.

\section{Submit your next manuscript to BioMed Central and take full advantage of:}

- Convenient online submission

- Thorough peer review

- No space constraints or color figure charges

- Immediate publication on acceptance

- Inclusion in PubMed, CAS, Scopus and Google Scholar

- Research which is freely available for redistribution

Submit your manuscript at www.biomedcentral.com/submit
C) Biomed Central 